\title{
On the Rainbow Turán number of paths
}

\author{
Beka Ergemlidze \\ Department of Mathematics \\ Central European University \\ Budapest, Hungary \\ beka.ergemlidze@gmail.com
}

\author{
Ervin Györi \\ Alfréd Rényi Institute of Mathematics \\ and \\ Central European University \\ Budapest, Hungary \\ gyori.ervin@renyi.mta.hu
}

\author{
Abhishek Methuku \\ Department of Mathematics \\ École Polytechnique Fédérale de Lausanne \\ Switzerland \\ abhishekmethuku@gmail.com
}

Submitted: May 25, 2018; Accepted: Dec 15, 2018; Published: Feb 8, 2019

(C) The authors. Released under the CC BY-ND license (International 4.0).

\begin{abstract}
Let $F$ be a fixed graph. The rainbow Turán number of $F$ is defined as the maximum number of edges in a graph on $n$ vertices that has a proper edge-coloring with no rainbow copy of $F$ (i.e., a copy of $F$ all of whose edges have different colours). The systematic study of such problems was initiated by Keevash, Mubayi, Sudakov and Verstraëte.

In this paper, we show that the rainbow Turán number of a path with $k+1$ edges is less than $(9 k / 7+2) n$, improving an earlier estimate of Johnston, Palmer and Sarkar.
\end{abstract}

Mathematics Subject Classifications: 05C35, 05C69

\section{Introduction}

Given a graph $F$, the maximum number of edges in a graph on $n$ vertices that contains no copy of $F$ is known as the Turán number of $F$, and is denoted by ex $(n, F)$. An edgecolored graph is called rainbow if all its edges have different colors. Given a graph $F$, the rainbow Turán number of $F$ is defined as the maximum number of edges in a graph on $n$ vertices that has a proper edge-coloring with no rainbow copy of $F$, and it is denoted by $\mathrm{ex}^{*}(n, F)$.

The systematic study of rainbow Turán numbers was initiated in [6] by Keevash, Mubayi, Sudakov and Verstraëte. Clearly, $\operatorname{ex}^{*}(n, F) \geq \operatorname{ex}(n, F)$. They determined $\operatorname{ex}^{*}(n, F)$ asymptotically for any non-bipartite graph $F$, by showing that $\operatorname{ex}^{*}(n, F)=$ $(1+o(1)) \operatorname{ex}(n, F)$. For bipartite $F$ with a maximum degree of $s$ in one of the parts, 
they proved $\operatorname{ex}^{*}(n, F)=O\left(n^{2-1 / s}\right)$. This matches the upper bound for the (usual) Turán numbers of such graphs.

Keevash, Mubayi, Sudakov and Verstraëte also studied the rainbow Turán problem for even cycles. More precisely, they showed that $\operatorname{ex}^{*}\left(n, C_{2 k}\right)=\Omega\left(n^{1+1 / k}\right)$ using the construction of large $B_{k}^{*}$-sets of Bose and Chowla [2]- it is conjectured that the same lower bound holds for $\operatorname{ex}^{*}\left(n, C_{2 k}\right)$ and is a well-known difficult open problem in extremal graph theory. They also proved a matching upper bound in the case of the six-cycle $C_{6}$, so it known that $\operatorname{ex}^{*}\left(n, C_{6}\right)=\Theta\left(n^{4 / 3}\right)=\operatorname{ex}\left(n, C_{6}\right)$. However, interestingly, they showed that $\operatorname{ex}^{*}\left(n, C_{6}\right)$ is asymptotically larger than $\operatorname{ex}\left(n, C_{6}\right)$ by a multiplicative constant. Recently, Das, Lee and Sudakov [3] showed that $\operatorname{ex}^{*}\left(n, C_{2 k}\right)=O\left(n^{1+\frac{\left(1+\epsilon_{k}\right) \ln k}{k}}\right)$, where $\epsilon_{k} \rightarrow 0$ as $k \rightarrow \infty$.

For an integer $k$, let $P_{k}$ denote a path of length $k$, where the length of a path is defined as the number of edges in it. Erdös and Gallai [4] proved that $\operatorname{ex}\left(n, P_{k+1}\right) \leq \frac{k}{2} n$; moreover, they showed that if $k+1$ divides $n$, then the unique extremal graph is the vertex-disjoint union of $\frac{n}{k+1}$ copies of $K_{k+1}$.

On the other hand, Keevash, Mubayi, Sudakov and Verstraëte [6] showed that in some cases, the rainbow Turán number of $P_{k}$ can be strictly larger than the usual Turán number of $P_{k}$ : Maamoun and Meyniel [7] gave an example of a proper coloring of $K_{2^{k}}$ containing no rainbow path with $2^{k}-1$ edges. By taking a vertex-disjoint union of such $K_{2^{k}}$ 's, Keevash et. al. showed that $\operatorname{ex}^{*}\left(n, P_{2^{k}-1}\right) \geq\left(\begin{array}{c}2^{k} \\ 2\end{array}\right)\left\lfloor\frac{n}{2^{k}}\right\rfloor=(1+o(1)) \frac{2^{k}-1}{2^{k}-2} \operatorname{ex}\left(n, P_{2^{k}-1}\right)-$ so $\operatorname{ex}^{*}\left(n, P_{2^{k}-1}\right)$ is not asymptotically equal to $\operatorname{ex}\left(n, P_{2^{k}-1}\right)$. They also mentioned that determining the asymptotic behavior of $\operatorname{ex}^{*}\left(n, P_{k+1}\right)$ is an interesting open problem, and stated the natural conjecture that the optimal construction is a disjoint union of cliques of size $c(k)$, where $c(k)$ is chosen as large as possible so that the cliques can be properly colored with no rainbow $P_{k+1}$. For $P_{4}$, this conjecture was disproved by Johnston, Palmer and Sarkar [5]: Since any properly edge-colored $K_{5}$ contains a rainbow $P_{4}$, and $K_{4}$ does not contain a $P_{4}$, the conjecture for $P_{4}$ would be that $\operatorname{ex}^{*}\left(n, P_{4}\right) \sim \frac{3 n}{2}$. But they show that in fact, ex* $\left(n, P_{4}\right) \sim 2 n$ by showing a proper edge-coloring of $K_{4,4}$ without no rainbow $P_{4}$, and then taking $\frac{n}{8}$ vertex-disjoint copies of $K_{4,4}$. For general $k$, they proved the following:

Theorem 1 (Johnston, Palmer and Sarkar [5]). For any positive integer $k$, we have

$$
\frac{k}{2} n \leq e x^{*}\left(n, P_{k+1}\right) \leq\left\lceil\frac{3 k+1}{2}\right\rceil n .
$$

We improve the above bound by showing the following:

Theorem 2. For any positive integer $k$, we have

$$
e x^{*}\left(n, P_{k+1}\right)<\left(\frac{9 k}{7}+2\right) n .
$$

Let us remark that using the ideas introduced in this paper, it is conceivable that the upper bound can be further improved (at the cost of making the proof very involved). However, it would be very interesting (and seems to be difficult) to prove an upper bound less than $k n$ or construct an example with $k n$ edges.

We give a construction which shows that $\operatorname{ex}^{*}\left(n, P_{2^{k}}\right)>\operatorname{ex}\left(n, P_{2^{k}}\right)$ for any $k \geq 2$. 
Construction. Let us first show a proper edge-coloring of $K_{2^{k}, 2^{k}}$ (a complete bipartite graph with parts $A$ and $B$, each of size $2^{k}$ ) with no rainbow $P_{2^{k}}$. The vertices of $A$ and $B$ are both identified with the vectors $\mathbb{F}_{2}^{k}$. Each edge $u v$ with $u \in A$ and $v \in B$ is assigned the color $c(u v):=u-v$. Clearly this gives a proper edge-coloring of $K_{2^{k}, 2^{k}}$. Moreover, if it contains a rainbow path $v_{0} v_{1} \ldots v_{2^{k}}$ then such a path must use all of the colors from $\mathbb{F}_{2}^{k}$. Therefore $\sum_{i=0}^{2^{k}-1} c\left(v_{i} v_{i+1}\right)=0$. On the other hand, $\sum_{i=0}^{2^{k}-1} c\left(v_{i} v_{i+1}\right)=$ $\sum_{i=0}^{2^{k}-1}\left(v_{i}-v_{i+1}\right)=v_{0}-v_{2^{k}}$. Thus, $v_{0}-v_{2^{k}}=0$. But notice that since the length of the path $v_{0} v_{1} \ldots v_{2^{k}}$ is even, its terminal vertices $v_{0}$ and $v_{2^{k}}$ are either both in $A$ or they are both in $B$. So they could not have been identified with the same vector in $\mathbb{F}_{2}^{k}$, a contradiction. Taking a vertex-disjoint union of such $K_{2^{k}, 2^{k}}$ 's we obtain that $\operatorname{ex}^{*}\left(n, P_{2^{k}}\right) \geq\left(2^{k}\right)^{2}\left\lfloor n / 2^{k+1}\right\rfloor=(1+o(1)) \frac{2^{k}}{2^{k}-1} \operatorname{ex}\left(n, P_{2^{k}}\right)$.

Remark. This construction provides a counterexample to the above mentioned conjecture of Keevash, Mubayi, Sudakov and Verstraëte [6] whenever the largest clique that can be properly colored without a rainbow $P_{2^{k}}$ has size $2^{k}$. This is the case for $k=2$, as noted before. The question of determining whether this is the case for any $k \geq 3$ remains an interesting open question (see [1] for results in this direction).

Overview of the proof and organization. Let $G$ be a graph which has a proper edge-coloring with no rainbow $P_{k+1}$. By induction on the length of the path, we assume there is a rainbow path $v_{0} v_{1} \ldots v_{k}$ in $G$. Roughly speaking, we will show that the sum of degrees of the terminal vertices of the path, $v_{0}$ and $v_{k}$ is small. Our strategy is to find a set of distinct vertices $M:=\left\{a_{1}, b_{1}, a_{2}, b_{2}, \ldots, a_{m}, b_{m}\right\} \subseteq\left\{v_{0}, v_{1}, \ldots, v_{k}\right\}$ (whose size is as large as possible) such that for each $1 \leq i \leq m$, there is a rainbow path $P$ of length $k$ with $a_{i}$ and $b_{i}$ as terminal vertices and $V(P)=\left\{v_{0}, v_{1}, \ldots, v_{k}\right\}$; then we show that there are not many edges of $G$ incident to the vertices of $M$, which will allow us to delete the vertices of $M$ from $G$ and apply induction. To this end, we define the set $T \subseteq\left\{v_{0}, v_{1}, \ldots, v_{k}\right\}$ as the set of all vertices $v \in\left\{v_{0}, v_{1}, \ldots, v_{k}\right\}$ where $v$ is a terminal vertex of some rainbow path $P$ with $V(P)=\left\{v_{0}, v_{1}, \ldots, v_{k}\right\}$; we call $T$ the set of terminal vertices. We will then find $M$ as a subset of $T$; moreover, it will turn out that if the size of $T$ is large, then the size of $M$ is also large - therefore, the heart of the proof lies in showing that $T$ is large.

In Section 2.1, we introduce the notation and prove some basic claims. Using these claims, in Section 2.2, we will show that $T$ is large (i.e., that there are many terminal vertices). Then in Section 2.3 we will find the desired subset $M$ of $T$ (which has few edges incident to it).

\section{Proof of Theorem 2}

Let $G$ be a graph on $n$ vertices, and suppose it has a proper edge-coloring $c: E(G) \rightarrow \mathbb{N}$ without a rainbow path of length $k+1$. Consider a longest rainbow path $P^{*}$ in $G$. We may suppose it is of length $k$, otherwise we are done by induction on $k$. For the base case $k=1$, notice that any path of length 2 , has to be a rainbow path. Thus $G$ can contain at most $\frac{n}{2}<\left(\frac{9}{7}+2\right) n$ edges, so we are done. 


\subsection{Basic claims and Notation}

In the rest of the paper, the degree of a vertex $v \in V(G)$ be denoted by $d(v)$.

Definition 3. Let $P^{*}=v_{0} v_{1} \ldots v_{k}$. Suppose the color of the edge $v_{i-1} v_{i}$ is $c\left(v_{i-1} v_{i}\right)=c_{i}$ for each $1 \leq i \leq k$. Let $L$ and $R$ denote the sets of colors of edges incident to $v_{0}$ and $v_{k}$ respectively. (Notice that since the edges of $G$ are colored properly, we have $|L|=d\left(v_{0}\right)$ and $|R|=d\left(v_{k}\right)$.)

We define the following subsets of $L, R$ and $\left\{c_{1}, c_{2}, \ldots, c_{k}\right\}$ corresponding to $P^{*}$.

- Let $L_{\text {out }}$ (respectively $R_{\text {out }}$ ) be the set of colors of the edges incident to $v_{0}$ (respectively $v_{k}$ ) and to a vertex outside $P^{*}$.

Note that $L_{\text {out }} \subseteq\left\{c_{1}, c_{2}, \ldots, c_{k}\right\}$ and $R_{\text {out }} \subseteq\left\{c_{1}, c_{2}, \ldots, c_{k}\right\}$, otherwise we can extend $P^{*}$ to a rainbow path longer than $k$ in $G$.

- Let $L_{\text {in }}=L \backslash L_{\text {out }}$ and $R_{\text {in }}=R \backslash R_{\text {out }}$.

- Let $L_{\text {old }}=L \cap\left\{c_{1}, c_{2}, \ldots, c_{k}\right\}$ and $L_{\text {new }}=L \backslash\left\{c_{1}, c_{2}, \ldots, c_{k}\right\}$. Similarly, let $R_{\text {old }}=$ $R \cap\left\{c_{1}, c_{2}, \ldots, c_{k}\right\}, R_{\text {new }}=R \backslash\left\{c_{1}, c_{2}, \ldots, c_{k}\right\}$.

- Let $S_{L}=\left\{c\left(v_{j-1} v_{j}\right)=c_{j} \mid v_{0} v_{j} \in E(G)\right.$ and $c\left(v_{0} v_{j}\right) \in L_{\text {new }}$ and $\left.2 \leq j \leq k\right\}$ and $S_{R}=\left\{c\left(v_{j} v_{j+1}\right)=c_{j+1} \mid v_{k} v_{j} \in E(G)\right.$ and $c\left(v_{k} v_{j}\right) \in R_{\text {new }}$ and $\left.0 \leq j \leq k-2\right\}$.

Notice that $\left|S_{L}\right|=\left|L_{\text {new }}\right|$ and $\left|S_{R}\right|=\left|R_{\text {new }}\right|$.

- Let $L_{\text {nice }}=L \cap S_{R}$ and let $R_{\text {nice }}=R \cap S_{L}$. (Note that since $L_{\text {nice }} \subseteq\left\{c_{1}, c_{2}, \ldots, c_{k}\right\}$, we have $L_{\text {nice }} \cap L_{\text {new }}=\emptyset$. Similarly $R_{\text {nice }} \cap R_{\text {new }}=\emptyset$.)

- Let $L_{\text {res }}=L_{\text {in }} \backslash\left(L_{\text {new }} \cup L_{\text {nice }}\right)=L_{\text {old }} \backslash\left(L_{\text {nice }} \cup L_{\text {out }}\right)$, and $R_{\text {res }}=R_{\text {in }} \backslash\left(R_{\text {new }} \cup R_{\text {nice }}\right)=$ $R_{\text {old }} \backslash\left(R_{\text {nice }} \cup R_{\text {out }}\right)$.

Notation. For convenience, we let $|L|=l$ and $|R|=r$. Moreover, let $\left|L_{\text {out }}\right|=l_{\text {out }},\left|L_{\text {old }}\right|=$ $l_{\text {old }},\left|L_{\text {nice }}\right|=l_{\text {nice }},\left|L_{\text {new }}\right|=l_{\text {new }}$ and $\left|R_{\text {out }}\right|=r_{\text {out }},\left|R_{\text {old }}\right|=r_{\text {old }},\left|R_{\text {nice }}\right|=r_{\text {nice }},\left|R_{\text {new }}\right|=$ $r_{\text {new }}$.

Note that

$$
d\left(v_{0}\right)=l_{\text {in }}+l_{\text {out }}=l_{\text {new }}+l_{\text {old }}=l
$$

and

$$
d\left(v_{k}\right)=r_{\text {in }}+r_{\text {out }}=r_{\text {new }}+r_{\text {old }}=r .
$$

Now we prove some inequalities connecting the quantities defined in Definition 3 for the path $P^{*}$.

Claim 4. $L_{\text {out }} \cap S_{R}=\emptyset=R_{\text {out }} \cap S_{L}$. This implies that $L_{\text {out }} \cap L_{\text {nice }}=\emptyset=R_{\text {out }} \cap R_{\text {nice }}$ (since $L_{\text {nice }} \subset S_{R}$ and $R_{\text {nice }} \subset S_{L}$ ).

Proof of Claim. Suppose for a contradiction that $L_{\text {out }} \cap S_{R} \neq \emptyset$. So there exists a vertex $w \notin\left\{v_{0}, v_{1}, \ldots, v_{k}\right\}$ such that $c\left(v_{k} v_{j}\right) \in R_{\text {new }}$ and $c\left(w v_{0}\right)=c\left(v_{j} v_{j+1}\right)$ for some $0 \leq j \leq$ $k-2$. Consider the path $v_{j+1} v_{j+2} \ldots v_{k} v_{j} v_{j-1} \ldots v_{0} w$. The set of colors of the edges in this path is $\left\{c_{1}, c_{2}, \ldots, c_{k}\right\} \backslash\left\{c\left(v_{j} v_{j+1}\right)\right\} \cup\left\{c\left(w v_{0}\right), c\left(v_{k} v_{j}\right)\right\}=\left\{c_{1}, c_{2}, \ldots, c_{k}\right\} \cup\left\{c\left(v_{k} v_{j}\right)\right\}$, so it is a rainbow path of length $k+1$ in $G$, a contradiction.

Similarly, by a symmetric argument, we have $R_{\text {out }} \cap S_{L}=\emptyset$. 
Claim 5. $l_{\text {out }} \leq k-r_{\text {new }}$ and $r_{\text {out }} \leq k-l_{\text {new }}$.

Proof of Claim. By Claim 4, $L_{\text {out }} \cap S_{R}=\emptyset$. Since both $L_{\text {out }}$ and $S_{R}$ are subsets of $\left\{c_{1}, c_{2}, \ldots, c_{k}\right\}$, this implies, $\left|L_{\text {out }}\right|=l_{\text {out }} \leq k-\left|S_{R}\right|=k-r_{\text {new }}$, as desired. Similarly, $r_{\text {out }} \leq k-l_{\text {new }}$.

We will prove Theorem 2 by induction on the number of vertices $n$. For the base cases, note that for all $n \leq k$, the number of edges is trivially at most

$$
\left(\begin{array}{l}
n \\
2
\end{array}\right) \leq \frac{k n}{2}<\left(\frac{9 k}{7}+2\right) n,
$$

so the statement of the theorem holds. If $d(v)<\frac{9 k}{7}+2$ for some vertex $v$ of $G$, then we delete $v$ from $G$ to obtain a graph $G^{\prime}$ on $n-1$ vertices. By induction hypothesis, the number of edges in $G^{\prime}$ is less than $\left(\frac{9 k}{7}+2\right)(n-1)$. So the total number of edges in $G$ is less than $\left(\frac{9 k}{7}+2\right) n$, as desired.

Therefore, from now on, we assume that for all $v \in V(G)$,

$$
d(v) \geq \frac{9 k}{7}+2
$$

Since $d\left(v_{0}\right)=l=l_{\text {old }}+l_{\text {new }}$ and $l_{\text {old }} \leq k$, we have that

$$
l_{\text {new }} \geq \frac{2 k}{7}+2
$$

Similarly,

$$
r_{\text {new }} \geq \frac{2 k}{7}+2
$$

Claim 6. We have

$$
l_{\text {nice }}+r_{\text {nice }} \geq \frac{4 k}{7}+4 .
$$

Proof of Claim. First notice that $L_{\text {res }} \cap S_{R}=\emptyset$. Indeed, by definition, $L_{\text {res }} \cap S_{R}=$ $\left(L_{\text {res }} \cap L\right) \cap S_{R}=L_{\text {res }} \cap\left(L \cap S_{R}\right)=L_{\text {res }} \cap L_{\text {nice }}=\emptyset$. Moreover, by Claim $4, L_{\text {out }} \cap S_{R}=\emptyset$. Therefore, we have $\left(L_{\text {res }} \cup L_{\text {out }}\right) \cap S_{R}=\emptyset$. Moreover, $\left(L_{\text {res }} \cup L_{\text {out }}\right) \cup S_{R} \subseteq\left\{c_{1}, c_{2}, \ldots, c_{k}\right\}$. Therefore, $l_{\text {res }}+l_{\text {out }} \leq k-\left|S_{R}\right|=k-r_{\text {new }}$. On the other hand, by definition, $l_{\text {res }}+l_{\text {out }} \geq$ $\left(l_{\text {in }}-l_{\text {new }}-l_{\text {nice }}\right)+l_{\text {out }}=l-l_{\text {new }}-l_{\text {nice }}$. So we have,

$$
l-l_{\text {new }}-l_{\text {nice }} \leq k-r_{\text {new }} .
$$

By a symmetric argument, we get

$$
r-r_{\text {new }}-r_{\text {nice }} \leq k-l_{\text {new }}
$$

Adding the above two inequalities and rearranging, we get $l+r-l_{\text {nice }}-r_{\text {nice }} \leq 2 k$, so

$$
l_{\text {nice }}+r_{\text {nice }} \geq l+r-2 k=d\left(v_{0}\right)+d\left(v_{k}\right)-2 k \geq \frac{4 k}{7}+4,
$$

as required. 


\subsection{Finding many terminal vertices}

Definition 7 (Set of terminal vertices). Let $T$ be the set of all vertices

$$
v \in\left\{v_{0}, v_{1}, v_{2}, \ldots, v_{k}\right\}
$$

such that $v$ is a terminal vertex of some rainbow path $P$ with $V(P)=\left\{v_{0}, v_{1}, v_{2}, \ldots, v_{k}\right\}$.

For convenience, we will denote the size of $T$ by $t$.

The next lemma yields a lower bound on the number of terminal vertices and is crucial to the proof of Theorem 2 .

Lemma 8. We have

$$
|T|=t \geq \frac{3 k}{7}+2
$$

The rest of this subsection is devoted to the proof of Lemma 8.

\section{Proof of Lemma 8}

Recall that $P^{*}=v_{0} v_{1} \ldots v_{k}$ and $c\left(v_{j} v_{j+1}\right)=c_{j}$. First we prove a simple claim.

Claim 9. We may assume $c\left(v_{0} v_{1}\right) \notin L_{\text {nice }}$ and $c\left(v_{k} v_{k-1}\right) \notin R_{\text {nice }}$. Moreover, if $v_{0} v_{k}$ is an edge of $G$, we can assume $c\left(v_{0} v_{k}\right) \notin L_{\text {new }} \cup R_{\text {new }}$.

Proof of Claim. First consider the case when $v_{0} v_{k}$ is an edge of $G$. If $c\left(v_{0} v_{k}\right) \in L_{\text {new }} \cup R_{\text {new }}$, then every vertex $v_{i} \in T$. Indeed, the path $v_{i} v_{i-1} v_{i-2} \ldots v_{0} v_{k} v_{k-1} \ldots v_{i+1}$ is a rainbow path with $v_{i}$ as a terminal vertex. Thus $|T|=k+1 \geq \frac{3 k}{7}+2$, and we are done. So we can assume $c\left(v_{0} v_{k}\right) \notin L_{\text {new }} \cup R_{\text {new }}$. This implies that $c\left(v_{0} v_{1}\right) \notin L_{\text {nice }}$ and $c\left(v_{k} v_{k-1}\right) \notin R_{\text {nice }}$, because $c\left(v_{0} v_{1}\right) \notin S_{R}$ and $c\left(v_{k} v_{k-1}\right) \notin S_{L}$.

Now if $v_{0} v_{k}$ is not an edge of $G$, then again $c\left(v_{0} v_{1}\right) \notin S_{R}$ and $c\left(v_{k} v_{k-1}\right) \notin S_{L}$, so the claim follows.

Claim 10. If $v_{0} v_{i}$ is an edge such that $c\left(v_{0} v_{i}\right) \in L_{\text {new }}$ then $v_{i-1} \in T$.

Proof of Claim. Consider the path $v_{i-1} v_{i-2} \ldots v_{0} v_{i} v_{i+1} \ldots v_{k}$. Clearly it is a rainbow path of length $k$ in which $v_{i-1}$ is a terminal vertex.

Suppose $v_{0} v_{i}$ is an edge such that $c\left(v_{0} v_{i}\right) \in L_{\text {nice. }}$. Since $c\left(v_{0} v_{k}\right) \notin R_{\text {new }}$, by the definition of $L_{\text {nice }}$, there exists an integer $j$ (with $1 \leq j \leq k-2$ ) such that $c\left(v_{k} v_{j}\right) \in R_{\text {new }}$ and $c\left(v_{0} v_{i}\right)=c\left(v_{j} v_{j+1}\right)=c_{j}$.

Claim 11. If $c\left(v_{0} v_{i}\right) \in L_{\text {nice }}$ then $v_{i-1} \in T$ or $v_{i+1} \in T$.

Moreover, let $j$ be an integer (with $1 \leq j \leq k-2$ ) such that $c\left(v_{k} v_{j}\right) \in R_{\text {new }}$ and $c\left(v_{0} v_{i}\right)=c\left(v_{j} v_{j+1}\right)=c_{j}$.

If $j \geq i$, then $v_{i-1} \in T$, and if $j<i$ then $v_{i+1} \in T$.

Proof of Claim. Observe that since $c\left(v_{0} v_{i}\right) \in L_{\text {nice }} \subset S_{R}$, we have that $c\left(v_{k} v_{j}\right) \in R_{\text {new }}$ (by definition of $S_{R}$ ).

First let $j \geq i$. In this case consider the path $v_{i-1} v_{i-2} \ldots v_{0} v_{i} v_{i+1} \ldots v_{j} v_{k} v_{k-1} \ldots v_{j+1}$. It is easy to see that the set of colors of the edges in this path is $\left\{c_{1}, c_{2}, \ldots, c_{k}\right\} \backslash\left\{c_{i}\right\} \cup$ 
$\left\{c\left(v_{j} v_{k}\right)\right\}$. As $c\left(v_{j} v_{k}\right) \in R_{\text {new }}$, the path is rainbow with $v_{i-1}$ as a terminal vertex. So $v_{i-1} \in T$.

If $j<i$, then consider the path $v_{j+1} v_{j+2} \ldots v_{i} v_{0} v_{1} \ldots v_{j} v_{k} v_{k-1} \ldots v_{i+1}$. It is easy to see that the set of colors of the edges in this path is $\left\{c_{1}, c_{2}, \ldots, c_{k}\right\} \backslash\left\{c_{i+1}\right\} \cup\left\{c\left(v_{j} v_{k}\right)\right\}$, so the path is rainbow again, with $v_{i+1}$ as a terminal vertex. So $v_{i+1} \in T$.

By symmetry, one can see that the same arguments used in the proofs of Claim 10 and Claim 11, imply the following two statements.

Observation 12. If $v_{k} v_{i}$ is an edge such that $c\left(v_{k} v_{i}\right) \in R_{\text {new }}$ then $v_{i+1} \in T$.

If $c\left(v_{k} v_{i}\right) \in R_{\text {nice }}$ then $v_{i-1} \in T$ or $v_{i+1} \in T$.

Definition 13. Let $b^{\prime}>b$ be the largest two integers such that $c\left(v_{0} v_{b}\right) \in L_{\text {new }}$ and $c\left(v_{0} v_{b^{\prime}}\right) \in L_{\text {new }}$. Similarly, let $a^{\prime}<a$ be the smallest two integers such that $c\left(v_{k} v_{a^{\prime}}\right) \in R_{\text {new }}$ and $c\left(v_{k} v_{a}\right) \in R_{\text {new }}$.

Notation. For any integers, $0 \leq x \leq y \leq k$, let

$$
T^{x, y}=\left\{v_{i} \in T \mid x \leq i \leq y\right\},
$$

and $\left|T^{x, y}\right|=t^{x, y}$.

Notice that $t=t^{0, k}=2+t^{1, k-1}$, as $v_{0}$ and $v_{k}$ are both terminal vertices.

Now we will show that if $a>b$, then Lemma 8 holds. Suppose $a>b$. Then by the definition of $a$ and $b$, we have

$$
\mid\left\{i \mid 2 \leq i \leq b \text { and } c\left(v_{0} v_{i}\right) \in L_{\text {new }}\right\}|=| L_{\text {new }} \mid-1=l_{\text {new }}-1 .
$$

By Claim 10, we know that whenever $c\left(v_{0} v_{i}\right) \in L_{\text {new }}$, we have $v_{i-1} \in T$. This shows that $t^{1, b-1} \geq l_{\text {new }}-1$. Similarly, by a symmetric argument (using Observation 12), we get $t^{a+1, k-1} \geq r_{\text {new }}-1$. Therefore,

$$
t=2+t^{1, k-1}=2+t^{1, b-1}+t^{b, a}+t^{a+1, k-1} \geq 2+\left(l_{\text {new }}-1\right)+\left(r_{\text {new }}-1\right)=l_{\text {new }}+r_{\text {new }} .
$$

Now using (1) and (2), we have

$$
t=l_{\text {new }}+r_{\text {new }} \geq \frac{2 k}{7}+2+\frac{2 k}{7}+2=\frac{4 k}{7}+4,
$$

proving Lemma 8. Therefore, from now on, we always assume $a \leq b$.

Claim 14. If $c\left(v_{0} v_{i}\right) \in L_{\text {new }}$ or $c\left(v_{k} v_{i}\right) \in R_{\text {new }}$, and $a \leq i \leq b$, then $v_{i-1} \in T$ and $v_{i+1} \in T$.

Proof of Claim. First suppose $c\left(v_{0} v_{i}\right) \in L_{\text {new }}$. Then by Claim 10, $v_{i-1} \in T$. We want to show that $v_{i+1} \in T$.

Observe that if $i=a$, then by Claim 10 again, we have $v_{i+1} \in T$ because $v_{k} v_{i} \in R_{\text {new }}$. So let us assume $a<i$ and show that $v_{i+1} \in T$. Notice that there exists $a^{*} \in\left\{a, a^{\prime}\right\}$ (see Definition 13 for the definition of $a$ and $\left.a^{\prime}\right)$ such that $c\left(v_{0} v_{i}\right) \neq c\left(v_{a^{*}} v_{k}\right)$. Now consider the path $v_{a^{*}+1} v_{a^{*}+2} \ldots v_{i} v_{0} v_{1} \ldots v_{a^{*}} v_{k} v_{k-1} \ldots v_{i+1}$. The set of colors of the edges in this path are $\left\{c_{1}, c_{2}, \ldots, c_{k}\right\} \backslash\left\{c_{a^{*}+1}, c_{i+1}\right\} \cup\left\{c\left(v_{0} v_{i}\right), c\left(v_{a^{*}} v_{k}\right)\right\}$, and it is easy to check that all the colors are different, so the path is rainbow with $v_{i+1}$ as a terminal vertex.

Now suppose $c\left(v_{k} v_{i}\right) \in R_{\text {new }}$. Then a similar argument (using Observation 12) shows that $v_{i-1} \in T$ and $v_{i+1} \in T$ again, completing the proof of the claim. 
Now we introduce some helpful notation.

Notation. For any integers, $0 \leq x \leq y \leq k$, let

$$
\begin{aligned}
& L_{\text {nice }}^{x, y}=\left\{c\left(v_{0} v_{i}\right) \in L_{\text {nice }} \mid x \leq i \leq y\right\}, \\
& R_{\text {nice }}^{x, y}=\left\{c\left(v_{k} v_{i}\right) \in R_{\text {nice }} \mid x \leq i \leq y\right\}, \\
& L_{\text {new }}^{x, y}=\left\{c\left(v_{0} v_{i}\right) \in L_{\text {new }} \mid x \leq i \leq y\right\}, \\
& R_{\text {new }}^{x, y}=\left\{c\left(v_{k} v_{i}\right) \in R_{\text {new }} \mid x \leq i \leq y\right\},
\end{aligned}
$$

Moreover, let $\left|L_{\text {nice }}^{x, y}\right|=l_{\text {nice }}^{x, y},\left|R_{\text {nice }}^{x, y}\right|=r_{\text {nice }}^{x, y},\left|L_{\text {new }}^{x, y}\right|=l_{\text {new }}^{x, y},\left|R_{\text {new }}^{x, y}\right|=r_{\text {new }}^{x, y}$.

Note that by definition of $a$ and $b, l_{\text {new }}=l_{\text {new }}^{0, a-1}+l_{\text {new }}^{a, b}+1$ and $r_{\text {new }}=1+r_{\text {new }}^{a, b}+r_{\text {new }}^{b+1, k}$. Using Claim 9, for any integer $z$, we have the following:

$$
L_{\text {nice }}^{0, z}=L_{\text {nice }}^{2, z} \text { and } R_{\text {nice }}^{z, k}=R_{\text {nice }}^{z, k-2} \text {. }
$$

Moreover, by definition of $L_{\text {new }}$ and $R_{\text {new }}$, we have

$$
L_{\text {new }}^{0, z}=L_{\text {new }}^{2, z} \text { and } R_{\text {new }}^{z, k}=R_{\text {new }}^{z, k-2} .
$$

Informally speaking, Claim 11 and Claim 14 assert that each edge $e=v_{0} v_{i}$ such that $c\left(v_{0} v_{i}\right) \in L_{\text {new }} \cup L_{\text {nice }}$ "creates" a terminal vertex $x=v_{i-1} \in T$ or $x=v_{i+1} \in T$ (or sometimes both). Similarly, (using Observation 12) each edge $e=v_{k} v_{i}$ such that

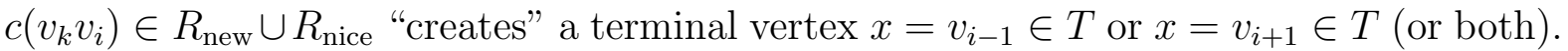
In the next two claims, by double counting the total number of such pairs $(e, x)$, we prove lower bounds on the number of terminal vertices in different ranges (i.e., $t^{0, a-1}, t^{b+1, k}$ and $\left.t^{a, b}\right)$, in terms of $l_{\text {new }}, r_{\text {new }}, l_{\text {nice }}$ and $r_{\text {nice }}$.

Claim 15. We have,

$$
t^{0, a-1} \geq \frac{1}{2}\left(l_{\text {nice }}^{0, a}+l_{\text {new }}^{0, a}+\frac{r_{\text {nice }}^{0, a}}{2}\right)
$$

and

$$
t^{b+1, k} \geq \frac{1}{2}\left(r_{\text {nice }}^{b, k}+r_{\text {new }}^{b, k}+\frac{l_{\text {nice }}^{b, k}}{2}\right)
$$

Proof of Claim. By Claim 11, and by the fact that there is only one $j$ such that $c\left(v_{k} v_{j}\right) \in$ $R_{\text {new }}^{0, a-1}$, it is easy to see that for all but at most one $i$, we have the following: if $c\left(v_{0} v_{i}\right) \in$ $L_{\text {nice }}^{0, a}=L_{\text {nice }}^{2, a}$ (equality here follows from (3)), then $v_{i-1} \in T^{1, a-1}$. So there are at least $l_{\text {nice }}^{2, a}-1$ pairs $\left(v_{0} v_{i}, x\right)$ such that $c\left(v_{0} v_{i}\right) \in L_{\text {nice }}^{2, a}$ and $x=v_{i-1} \in T^{1, a-1}$.

If $c\left(v_{0} v_{i}\right) \in L_{\text {new }}^{0, a}=L_{\text {new }}^{2, a}$ (equality here follows from (4)), then by Claim 10, $v_{i-1} \in$ $T^{1, a-1}$. So there are $l_{\text {new }}^{2, a}$ pairs $\left(v_{0} v_{i}, x\right)$ such that $c\left(v_{0} v_{i}\right) \in L_{\text {new }}^{2, a}$ and $x=v_{i-1} \in T^{1, a-1}$.

Adding the previous two bounds, the total number of pairs $\left(v_{0} v_{i}, x\right)$ such that $c\left(v_{0} v_{i}\right) \in$ $L_{\text {nice }}^{0, a} \cup L_{\text {new }}^{0, a}=L_{\text {nice }}^{2, a} \cup L_{\text {new }}^{2, a}$ and $x=v_{i-1} \in T^{1, a-1}$, is at least $l_{\text {nice }}^{2, a}-1+l_{\text {new }}^{2, a}$. This implies $t^{1, a-1} \geq l_{\text {nice }}^{2, a}-1+l_{\text {new }}^{2, a}$. Therefore, using that $v_{0}$ is also a terminal vertex, we have

$$
t^{0, a-1} \geq l_{\text {nice }}^{2, a}+l_{\text {new }}^{2, a} .
$$

If $c\left(v_{k} v_{i}\right) \in R_{\text {nice }}^{0, a-1}$, then by Observation 12, there is a vertex $x \in\left\{v_{i-1}, v_{i+1}\right\}$ such that $x \in T$. So the number of pairs $\left(v_{k} v_{i}, x\right)$ such that $c\left(v_{k} v_{i}\right) \in R_{\text {nice }}^{0, a-1}, x \in\left\{v_{i-1}, v_{i+1}\right\}$ 
and $x \in T$, is at least $r_{\text {nice }}^{0, a-1}$. By the pigeonhole principle, either the number of pairs $\left(v_{k} v_{i}, v_{i-1}\right)$ with $c\left(v_{k} v_{i}\right) \in R_{\text {nice }}^{0, a-1}, v_{i-1} \in T$, or the number of pairs $\left(v_{k} v_{i}, v_{i+1}\right)$ with $c\left(v_{k} v_{i}\right) \in R_{\text {nice }}^{0, a-1}, v_{i+1} \in T$, is at least $r_{\text {nice }}^{0, a-1} / 2$. In the first case, we get $t^{0, a-2} \geq r_{\text {nice }}^{0, a-1} / 2$ and in the second case, we get $t^{1, a} \geq r_{\text {nice }}^{0, a-1} / 2$. As $t^{0, a-1} \geq t^{0, a-2}$ and $t^{0, a-1} \geq t^{1, a}$, in both cases we have,

$$
t^{0, a-1} \geq \frac{r_{\text {nice }}^{0, a-1}}{2}
$$

Therefore, adding up (5) and (6), we get

$$
2 t^{0, a-1} \geq l_{\text {nice }}^{2, a}+l_{\text {new }}^{2, a}+\frac{r_{\text {nice }}^{0, a-1}}{2}=l_{\text {nice }}^{0, a}+l_{\text {new }}^{0, a}+\frac{r_{\text {nice }}^{0, a}}{2} .
$$

Note that the equality follows from (3), (4) and the fact that $r_{\text {nice }}^{0, a-1}=r_{\text {nice }}^{0, a}$ because $c\left(v_{k} v_{a}\right) \in R_{\text {new }}$. By a symmetric argument, we have

$$
2 t^{b+1, k} \geq r_{\text {nice }}^{b, k-2}+r_{\text {new }}^{b, k-2}+\frac{l_{\text {nice }}^{b+1, k}}{2}=r_{\text {nice }}^{b, k}+r_{\text {new }}^{b, k}+\frac{l_{\text {nice }}^{b, k}}{2}
$$

This finishes the proof of the claim.

Now we prove a lower bound on $t^{a, b}$.

\section{Claim 16.}

$$
t^{a, b} \geq \frac{1}{4}\left(l_{\text {nice }}^{a+1, b-1}+r_{\text {nice }}^{a+1, b-1}+2\left(l_{\text {new }}^{a+1, b}+r_{\text {new }}^{a, b-1}\right)\right) .
$$

Proof of Claim. Let us construct a set $S$ of pairs $(e, x)$ such that $e \in L_{\text {in }} \cup R_{\text {in }}$ and $x \in T$ with certain properties.

For every edge $e$ such that $c(e) \in L_{\text {nice }}^{a+1, b-1} \cup R_{\text {nice }}^{a+1, b-1}$, Claim 11 (and Observation 12) ensures that there is a vertex $x \in\left\{v_{i-1}, v_{i+1}\right\}$ such that $x \in T$ (in particular, $x \in T^{a, b}$ ). Add all such pairs $(e, x)$ to $S$. Therefore, the number of pairs $(e, x)$ added to $S$ so far, is $l_{\text {nice }}^{a+1, b-1}+r_{\text {nice }}^{a+1, b-1}$.

For every edge $e$ such that $c(e) \in L_{\text {new }}^{a+1, b} \cup R_{\text {new }}^{a, b-1}$, we have both $v_{i-1}, v_{i+1} \in T$ by Claim 14 ; we add both the pairs $\left(e, v_{i-1}\right)$ and $\left(e, v_{i+1}\right)$ to $S$. Therefore the number of pairs $(e, x)$ added to $S$ in this step is $2\left(l_{\text {new }}^{a+1, b}+r_{\text {new }}^{a, b-1}\right)$. Thus,

$$
|S|=l_{\text {nice }}^{a+1, b-1}+r_{\text {nice }}^{a+1, b-1}+2\left(l_{\text {new }}^{a+1, b}+r_{\text {new }}^{a, b-1}\right) .
$$

Note that all the pairs $(e, x)$ in $S$ are such that $x \in T^{a, b}$. Moreover, for each $x \in T^{a, b}$, there are at most four pairs $(e, x)$ in $S$. Therefore, we have

$$
4 t^{a, b} \geq|S| \geq l_{\text {nice }}^{a+1, b-1}+r_{\text {nice }}^{a+1, b-1}+2\left(l_{\text {new }}^{a+1, b}+r_{\text {new }}^{a, b-1}\right),
$$

finishing the proof of the claim.

By Claim 15 and Claim 16, we have

$$
\begin{aligned}
2\left(2 t^{0, a-1}+2 t^{b+1, k}\right)+4 t^{a, b} & \geq 2\left(l_{\text {nice }}^{0, a}+l_{\text {new }}^{0, a}+\frac{r_{\text {nice }}^{0, a}}{2}+r_{\text {nice }}^{b, k}+r_{\text {new }}^{b, k}+\frac{l_{\text {nice }}^{b, k}}{2}\right) \\
+l_{\text {nice }}^{a+1, b-1} & +r_{\text {nice }}^{a+1, b-1}+2\left(l_{\text {new }}^{a+1, b}+r_{\text {new }}^{a, b-1}\right) .
\end{aligned}
$$


This implies,

$$
4 t \geq l_{\text {nice }}+r_{\text {nice }}+2 l_{\text {new }}^{0, b}+2 r_{\text {new }}^{a, k}+l_{\text {nice }}^{0, a}+r_{\text {nice }}^{b, k} .
$$

By the definition of $a$ and $b, l_{\text {new }}^{0, b}=l_{\text {new }}-1$ and $r_{\text {new }}^{a, k}=r_{\text {new }}-1$. So, we get

$$
\begin{gathered}
4 t \geq l_{\text {nice }}+r_{\text {nice }}+2 l_{\text {new }}+2 r_{\text {new }}+l_{\text {nice }}^{0, a}+r_{\text {nice }}^{b, k}-4 \\
\geq l_{\text {nice }}+r_{\text {nice }}+2\left(l_{\text {new }}+r_{\text {new }}\right)-4 .
\end{gathered}
$$

Now by Claim 6 and inequalities (1) and (2), we get that

$$
4 t \geq \frac{4 k}{7}+4+2\left(\frac{2 k}{7}+2+\frac{2 k}{7}+2\right)-4=\frac{12 k}{7}+8 .
$$

Therefore,

$$
t \geq \frac{3 k}{7}+2
$$

completing the proof of Lemma 8.

\subsection{Finding a large subset of vertices with few incident edges}

Now we define an auxiliary graph $H$ with the vertex set $V(H)=T$ and edge set $E(H)$ such that $a b \in E(H)$ if and only if there is a rainbow path $P$ in $G$ with $a$ and $b$ as its terminal vertices and $V(P)=V\left(P^{*}\right)=\left\{v_{0}, v_{1}, \ldots, v_{k}\right\}$.

Claim 17. The degree of every vertex $u$ in $H$ is at least $2 k / 7+2$.

Proof of Claim. As $u \in V(H)=T, u$ is a terminal vertex. So there is a rainbow path $P=u_{0} u_{1} \ldots u_{k}$ in $G$ such that $u_{0}=u$ and $\left\{u_{0}, u_{1}, \ldots, u_{k}\right\}=\left\{v_{0}, v_{1}, \ldots, v_{k}\right\}$. We define the sets $L, R, L_{\text {new }}, R_{\text {new }}$ corresponding to $P$ in the same way as we did for $P^{*}$ (in Definition 3). Moreover, since $P^{*}$ was defined as an arbitrary rainbow path of length $k$, (2) holds for $P$ as well - i.e., $\left|R_{\text {new }}\right|=r_{\text {new }} \geq 2 k / 7+2$. We claim that if $u_{k} u_{j}$ is an edge in $G$ such that $c\left(u_{k} u_{j}\right) \in R_{\text {new }}$, then $u u_{j+1} \in E(H)$. Indeed, consider the path $u_{0} u_{1} \ldots u_{j} u_{k} u_{k-1} \ldots u_{j+1}$. This is clearly a rainbow path with terminal vertices $u=u_{0}$ and $u_{j+1}$. So $u$ and $u_{j+1}$ are adjacent in $H$, as required. This shows that degree of $u$ in $H$ is at least $r_{\text {new }} \geq 2 k / 7+2$, as desired.

Size of a matching is defined as the number of edges in it. The following proposition is folklore.

Proposition 18. Any graph $G$ with minimum degree $\delta(G)$ has a matching of size

$$
\min \left\{\delta(G),\left\lfloor\frac{V(G)}{2}\right\rfloor\right\} .
$$

We know that $\delta(H) \geq \frac{2 k}{7}+2$ by Claim 17. Moreover $|V(H)|=|T|=t$. So applying Proposition 18 to the graph $H$ and using Lemma 8, we obtain that the graph $H$ contains a matching $M$ of size

$$
m:=\min \left\{\frac{2 k}{7}+2,\left\lfloor\frac{t}{2}\right\rfloor\right\} \geq \frac{3 k}{14} .
$$

Let the edges of $M$ be $a_{1} b_{1}, a_{2} b_{2}, \ldots, a_{m} b_{m}$. Moreover, let

$$
n_{i}=\mid\left\{x y \mid x y \notin E(G), x \in\left\{a_{i}, b_{i}\right\} \text { and } y \in\left\{v_{0}, v_{1}, v_{2}, \ldots, v_{k}\right\} \backslash\left\{a_{i}, b_{i}\right\}\right\} \mid .
$$


Claim 19. The number of edges in the subgraph of $G$ induced by $M$ is

$$
|E(G[M])| \geq\left(\begin{array}{c}
2 m \\
2
\end{array}\right)-\left(\sum_{i=1}^{m} \frac{n_{i}}{2}+m\right)=2 m^{2}-2 m-\sum_{i=1}^{m} \frac{n_{i}}{2} .
$$

Proof of Claim. Note that the sum $\sum_{i} n_{i}$ counts each pair $x y \notin E(G)$ with $x, y \in V(M)$ exactly twice unless $x y=a_{i} b_{i}$ for some $i$. Therefore, the number of pairs $x y \notin E(G)$ in the subgraph of $G$ induced by $M$ is at most $\sum_{i} \frac{n_{i}}{2}+m$. Thus the number of edges of $G$ in the subgraph induced by $M$ is at least $\left(\begin{array}{c}2 m \\ 2\end{array}\right)-\left(\sum_{i} \frac{n_{i}}{2}+m\right)$, which implies the desired claim.

Claim 20. The sum of degrees of $a_{i}$ and $b_{i}$ in $G$ is at most $3 k-\frac{n_{i}}{2}$.

Proof of Claim. Since $a_{i} b_{i}$ is an edge in the auxiliary graph $H$, there is a rainbow path $P=u_{0} u_{1} \ldots u_{k}$ in $G$ such that $u_{0}=a_{i}, u_{k}=b_{i}$ and $\left\{u_{0}, u_{1}, \ldots, u_{k}\right\}=\left\{v_{0}, v_{1}, \ldots, v_{k}\right\}$. We define the sets $L, R, L_{\text {in }}, R_{\text {in }}, L_{\text {out }}, R_{\text {out }}, L_{\text {new }}, R_{\text {new }}$ and the numbers $l, r, l_{\text {in }}, r_{\text {in }}, l_{\text {out }}, r_{\text {out }}$, $l_{\text {new }}, r_{\text {new }}$ corresponding to $P$ in the same way as we did for $P^{*}$ (in Definition 3). Therefore, degree of $a_{i}$ is $l \leq l_{\text {new }}+k$. Similarly, degree of $b_{i}$ is at most $r_{\text {new }}+k$. So the sum of degrees of $a_{i}$ and $b_{i}$ in $G$ is at most

$$
2 k+l_{\text {new }}+r_{\text {new }} .
$$

On the other hand, the sum of degrees of $a_{i}$ and $b_{i}$ in $G$ is $l+r=l_{\text {in }}+l_{\text {out }}+r_{\text {in }}+r_{\text {out }}$. By Claim 5 , this is at most $\left(l_{\text {in }}+r_{\text {in }}\right)+k-r_{\text {new }}+k-l_{\text {new }}=\left(l_{\text {in }}+r_{\text {in }}\right)+2 k-l_{\text {new }}-r_{\text {new }}$. Moreover, it is easy to see that $l_{\text {in }}+r_{\text {in }} \leq 2 k-n_{i}$ by the definition of $n_{i}$. Therefore, the sum of degrees of $a_{i}$ and $b_{i}$ in $G$ is at most

$$
2 k-n_{i}+2 k-l_{\text {new }}-r_{\text {new }} .
$$

Adding up (8) and (9) and dividing by 2, we get that the sum of degrees of $a_{i}$ and $b_{i}$ in $G$ is at most

as desired.

$$
\frac{\left(2 k+2 k-n_{i}+2 k\right)}{2}=\frac{\left(6 k-n_{i}\right)}{2}=3 k-\frac{n_{i}}{2},
$$

The sum $\sum_{i=1}^{m}\left(d\left(a_{i}\right)+d\left(b_{i}\right)\right)$ counts each edge in the subgraph of $G$ induced by $M$ exactly twice (note that here $d(v)$ denotes the degree of the vertex $v$ in $G$ ). Therefore, the number of edges of $G$ incident to the vertices of $M$ is at most $\sum_{i=1}^{m}\left(d\left(a_{i}\right)+d\left(b_{i}\right)\right)-$ $|E(G[M])|$. Now using Claim 19 and Claim 20, the number of edges of $G$ incident to the vertices of $M$ is at most

$$
\sum_{i=1}^{m}\left(3 k-\frac{n_{i}}{2}\right)-\left(2 m^{2}-2 m-\sum_{i=1}^{m} \frac{n_{i}}{2}\right)=3 k m-2 m^{2}+2 m=(3 k+2-2 m) m .
$$

Now by (7), this is at most

$$
(3 k+2-2 m) m \leq\left(3 k+2-2\left(\frac{3 k}{14}\right)\right) m=\left(\frac{9 k}{7}+1\right) 2 m<\left(\frac{9 k}{7}+2\right) 2 m .
$$

We may delete the vertices of $M$ from $G$ to obtain a graph $G^{\prime}$ on $n-2 m$ vertices. By induction hypothesis, $G^{\prime}$ contains less than $\left(\frac{9 k}{7}+2\right)(n-2 m)$ edges. Therefore, $G$ contains less than

$$
\left(\frac{9 k}{7}+2\right) 2 m+\left(\frac{9 k}{7}+2\right)(n-2 m)=\left(\frac{9 k}{7}+2\right) n
$$

edges, as desired. This completes the proof of Theorem 2 . 


\section{Acknowledgements}

We are grateful to an anonymous referee for carefully reading our paper, and providing many helpful comments.

The research of the authors is partially supported by the National Research, Development and Innovation Office ANKFIH grant K116769. Additionally, the research of the second author is supported by the grant SNN117879.

\section{References}

[1] N. Alon, A. Pokrovskiy and B. Sudakov. Random subgraphs of properly edgecoloured complete graphs and long rainbow cycles. Israel Journal of Mathematics (2017) 222.1, 317-331.

[2] R.C. Bose and S. Chowla. Theorems in the additive theory of numbers. Comment. Math. Helv. (1962/1963) 37, 141-147.

[3] S. Das, C. Lee and B. Sudakov. Rainbow Turán problem for even cycles. European Journal of Combinatorics (2013) 34, 905-915.

[4] P. Erdős and T. Gallai. On maximal paths and circuits of graphs. Acta Mathematica Academiae Scientiarum Hungaricae (1959) 10, 337-356.

[5] D. Johnston, C. Palmer and A. Sarkar. Rainbow Turán Problems for Paths and Forests of Stars. The Electronic Journal of Combinatorics (2017) 24(1), \#P1.34.

[6] P. Keevash, D. Mubayi, B. Sudakov and J. Verstraëte. Rainbow Turán problems. Combinatorics, Probability and Computing (2007) 16, 109-126.

[7] M. Maamoun and H. Meyniel. On a problem of G. Hahn about coloured Hamiltonian paths in $K_{2^{t}}$. Discrete Mathematics (1984) 51, 213-214. 